\title{
Analysis of Adaptive and Advanced Speckle Filters on SAR Data
}

\author{
Arundhati Misra ${ }^{1}$, Dhwani Ajmera ${ }^{2}$ \\ ${ }^{I}$ (SAC, ISRO, Ahmedabad, India) \\ ${ }^{2}$ (Institute of Technology, EC Department, NIRMA University, India)
}

\begin{abstract}
Synthetic Aperture RADAR(SAR) images get inherently affected by speckle noise which is multiplicative in nature. This noise affects the image spatial statistics and properties. Over the past several years, many SAR denoising algorithms have been developed to reduce speckle noise. Some of the standard speckle filters are Gamma MAP, Lee, Frost and Kuan filters. Further, these have also been modified to obtain better results after filtering, than their original counterparts. Apart from the standard speckle filters, advanced SAR filters like Block Matching 3 Dimensional (BM3D) are also present. In this paper several standard as well as advanced speckle filters have been analyzed and compared. For comparison, Quality Assessment has been performed where the filtered images are compared to each other using parameters like Radiometric Resolution and others. These parameters help to distinguish the performance of the filters on basis of signal strength, speckle reduction, mean preservation and edge and feature preservation. In the paper, radiometric resolution, speckle index and mean preservation index will be used to analyze among the performance of the filters.
\end{abstract}

Keywords: BM3D, Frost, Gamma MAP, Kuan, Lee, Quality Assessment, SAR, Speckle

\section{Introduction}

Speckle noise is granular noise that inherently affects RADAR, SAR, medical ultrasound and other such applications. Speckle noise is perceived to be multiplicative in nature as a result of which it affects the spatial statistics of the image. Further it reduces the image contrast and makes it difficult to interpret the image accurately. Speckle noise can be classified in 2 ways - one by k distribution and the other by Rayleigh distribution. In free space, speckle is simply considered as an infinite sum of independent, identical phasors with random amplitude and phase[1].Speckle noise can be reduced in 2 ways - one by multi-look techniques where several independent single look images are formed, these are summed and then averaged to obtain multilook images[2]. The other method is the use of speckle filters where filtering operation is performed post the image formation. Adaptive filters most commonly used are Gamma MAP, Lee, Frost and Kuan. Filters like Gamma MAP, Enhanced Lee, Enhanced Frost and Enhanced Kuan differentiate the image into 3 regions which are homogeneous, heterogeneous and strong scattering and then perform the filtering operation. Homogeneous regions are uniform regions where the statistical parameters are similar whereas heterogeneous regions have large differences in their statistical parameters. One way to distinguish such regions in an image is by considering coefficient of variation. Coefficient of variation is the ratio of the local standard deviation to the local mean.

\section{Adaptive Filters}

Filtering algorithms like Gamma MAP, Lee, Frost, Kuan and their modified versions come under this category as the filtering operation makes use of the statistical properties of the image, hence they are adaptive in nature. Filters like Median filter are non adaptive as they do not make use of any statistical properties of the image.

\subsection{Gamma MAP Filter}

This filter assumes Gaussian distribution of the speckle noise. The performance of the MAP filter is enhanced by fixing a minimum value of coefficient of variation for better speckle smoothing and an upper limit for texture or point target preservation. Thus if the coefficient of variation is lesser than the minimum value then the region is termed to be homogeneous, greater than the upper limit, then it is termed to be strong scattering region and if otherwise, then it is termed to be heterogeneous region[3]. For homogeneous regions, the estimated pixel value is

$\mathrm{D}=\check{\mathrm{R}}$ where $\check{\mathrm{R}}$ is the local mean value

For strong scattering regions,

$$
\mathrm{D}=\mathrm{R}
$$

For heterogeneous regions,

$$
\mathrm{D}=((\mathrm{k} \check{\mathrm{R}})+\sqrt{\mathrm{d}}) /(2 \alpha)
$$


$\mathrm{k}=\alpha-\mathrm{N}-1, \mathrm{~N}$ is the Number of Looks

$\alpha=\left(1+\mathrm{C}_{-} \mathrm{si}^{2}\right) /\left(\mathrm{C}_{-} \mathrm{v}^{2}-\mathrm{C}_{-} \mathrm{si}^{2}\right)$

C_si is the standard speckle index

$\mathrm{C} \_\mathrm{V}$ is the coefficient of variation $\mathrm{d}=\breve{\mathrm{R}}^{2} \mathrm{k}^{2}+4 \alpha \mathrm{N} \check{\mathrm{R}} \mathrm{R}$

The Gamma MAP filter is most commonly used among the standard speckle filters because of its desirable results.

\subsection{Lee Filter}

The Gamma MAP filter is most commonly used among the standard speckle filters because of its desirable results.

$$
\begin{aligned}
& \mathrm{D}=\breve{\mathrm{R}}+\mathrm{W}(\mathrm{R}-\check{\mathrm{R}}) \\
& \mathrm{W}=1-\mathrm{C} \_\mathrm{si}^{2} / \mathrm{C} \mathrm{v}^{2}
\end{aligned}
$$

Lee filter is able to suppress noise effectively in homogeneous regions but does not perform well in heterogeneous regions.

\subsection{Enhanced Lee Filter}

Lopes modified Lee filter to develop the Enhanced Lee filter which like Gamma MAP segregates the image into different regions and then performs filtering. For homogeneous and strong scattering regions, the estimated pixel value is same as that of Gamma MAP filter. For heterogeneous regions, the pixel value is

$$
\begin{aligned}
& \mathrm{D}=\breve{\mathrm{R}} \mathrm{W}+\mathrm{R}(1-\mathrm{W}) \\
& \mathrm{W}=\exp \left(-\mathrm{K} \_\mathrm{d}\left(\mathrm{C}_{-} \mathrm{V}-\mathrm{C} \_ \text {si }\right) /\left(\mathrm{C} \_ \text {max-C_v }\right)\right)
\end{aligned}
$$

$\mathrm{K} \_\mathrm{d}$ is called the Damping Factor

Enhanced Lee performs better than Lee filter as it aims to improve the ability of preserving edges in the image[7].

\subsection{Frost Filter}

The Frost filter is similar to the Lee filter and is based on the Minimum Mean Square Error (MMSE) criteria[5]. The estimated pixel in Frost Filter is equal to:-

$$
\begin{aligned}
& \mathrm{D}=\left(\mathrm{R}_{1} \mathrm{~W}_{1}+\mathrm{R}_{2} \mathrm{~W}_{2}+\ldots \mathrm{R}_{\mathrm{N}} \mathrm{W}_{\mathrm{N}}\right) /\left(\mathrm{W}_{1}+\mathrm{W}_{2} \ldots \mathrm{W}_{\mathrm{N}}\right) \\
& \mathrm{W}=\exp (-\mathrm{A} \mathrm{T}) \\
& \mathrm{A}=\mathrm{K} \text { _d C_V }
\end{aligned}
$$

$\mathrm{T}$ is called Euclidean distance [7]. The Frost filter behaves as a mean filter in uniform or homogeneous regions and as a high pass filter in high contrast regions.

\subsection{Enhanced Frost Filter}

The Enhanced Frost filter, being similar to the Enhanced Lee and Gamma MAP filter segregates the image into the 3 different regions of homogeneity, heterogeneity and strong scatter before performing the filtering operation. For homogeneous and strong scattering regions, the estimated pixel value is same as that of Gamma MAP and Enhanced Lee filters[6][8]. For heterogeneous regions, the pixel value is

$$
\begin{aligned}
& \mathrm{D}=\left(\mathrm{R}_{1} \mathrm{~W}_{1}+\mathrm{R}_{2} \mathrm{~W}_{2}+\ldots \mathrm{R}_{\mathrm{N}} \mathrm{W}_{\mathrm{N}}\right) /\left(\mathrm{W}_{1}+\mathrm{W}_{2} \ldots \mathrm{W}_{\mathrm{N}}\right) \\
& \mathrm{W}=\exp (-\mathrm{A} \mathrm{T}) \\
& \mathrm{A}=\mathrm{K} \text { _d }\left(\mathrm{C}_{-} \mathrm{V}-\mathrm{C} \_ \text {si }\right) /(\mathrm{C} \text { _max-C_v })
\end{aligned}
$$

Enhanced Frost filter, similar to Enhanced Lee aims to preserve edges and it does so more effectively compared to Frost filter.

\subsection{Kuan Filter}

Kuan filter is also similar to the Lee filter with the difference being in the weight that is considered for the target pixel. The estimated pixel equation is same as that of the Lee filter as it has been derived from the Lee filter[6]. The weight factor is:-

$$
\mathrm{W}=\left(1-\mathrm{C}_{-} \mathrm{si}^{2} / \mathrm{C} \_\mathrm{v}^{2}\right) /\left(1+\mathrm{C} \_\mathrm{si}^{2}\right)
$$

It is considered to be more accurate than Lee filter due to the fact that no approximation is required in the total derivation [6].

\section{Advanced Filters}

Advanced Filters like BM3D make use of more than 1 method to minimize noise. They tend to produce better results compared to the standard speckle filters. 


\subsection{Block Matching 3 Dimensional Filter}

Block Matching 3 Dimensional (BM3D) filter is one of the most advanced and commonly used filters for image denoising. The basic BM3D algorithm considers noise to be additive in nature. The BM3D algorithm consists of 2 major steps, the output of one which is then taken as the input of the next step. The output of the first filtering process results in the production of the basic estimate, this basic estimate is then used as the input to the next filtering process which produces the final estimate or the final filtered image. The BM3D algorithm consists of 2 major steps. These are[9][10]

1. The first step produces the basic estimate by use of hard thresholding. The hard thresholding is used during the collaborative filtering process. Only the noisy image is considered as the input along with the hard thresholding parameters.

2. The second step produces the final estimate by using Wiener filter. The basic estimate and noisy image are taken in as inputs along with the Wiener filter parameters.

The parameters for hard thresholding and Wiener filtering which are pre-set rely heavily on the standard deviation of noise. Further depending on the value of the standard deviation, the parameters differ. Also the 2D transform used in case of Wiener filtering can be either DCT or DST. BM3D can also be used with other methods to produce better results such as BM3D-SAPCA, where the technique of BM3D is combined with Shape Adaptive Principal Component Analysis (SAPCA). The 2D transform used is a Shape Adaptive DCT (SA-DCT), which makes the algorithm adaptive in nature compared to the conventional BM3D algorithm.

\subsection{Wavelet Based Filter}

Wavelet based filtering implies the use of wavelet families like Daubechies, Symlets, Coiflets or any other for the purpose of denoising. In this paper, Daubechies 2, thus 4 coefficient are used.Thedecom positionprocessisdone3times, thus multiple levels decomposition and reconstruction has been done. Also to reduce noise, thresholding has been performed. Soft or hard thresholding are the common thresholding methods that are implemented to reduce noise. In this paper soft thresholding has been performed. Further the threshold selected is $4 *$ sigma, where sigma refers to the individual standard deviation values of the 4 sub bands which are LH, HL and HH. The DC component LL is passed through without any thresholding [11].

\section{Quality Assessment}

It is extremely difficult to distinguish among the various speckle filters visually. To know if a particular filtering algorithm is truly reliable, effective and consistent, quality assessment of the despeckle SAR image is needed. Statistical analysis like computation of the mean and variance doesn't always help to identity the most effective filter[12]. In this paper, 3 parameters will be used to compare among the various filters.

\subsection{Radiometric Resolution}

The radiometric resolution mathematically is the reciprocal of signal to noise ratio. Thus higher the value of SNR, lower the value of radiometric resolution which is desirable. SNR in case of SAR images is computed as the ratio of the mean of the image to the standard deviation of the same. Radiometric resolution is implemented as[13]:-

$$
\gamma=10 \log (1+1 / \mathrm{SNR})
$$

Lower values of radiometric resolution are desirable as that means the SNR values will be higher. Higher values of SNR are resulted in when the standard deviation values are lesser.

\subsection{Speckle Index}

Speckle Index, like the Coefficient of Variation, is defined as the ratio of standard deviation to the mean of the image. The difference being, the image to be considered is the filtered image only. Hence, lower the standard deviation, the better and thus lesser the speckle index.

\subsection{Mean Preservation Index}

The mean of the filtered image should not vary much with reference to the raw data. This parameter helps to evaluate the mean preservation properties[12]. It can be defined as the ratio of the absolute difference between the mean of the noisy image and the mean of the filtered image to the mean of the noisy image. Lower values of MPI indicate good mean preservation by that particular filter. 


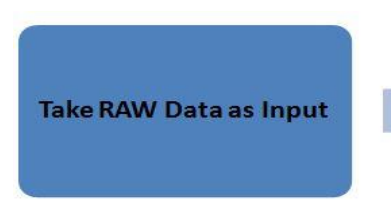

\section{Methodology and Data Set}
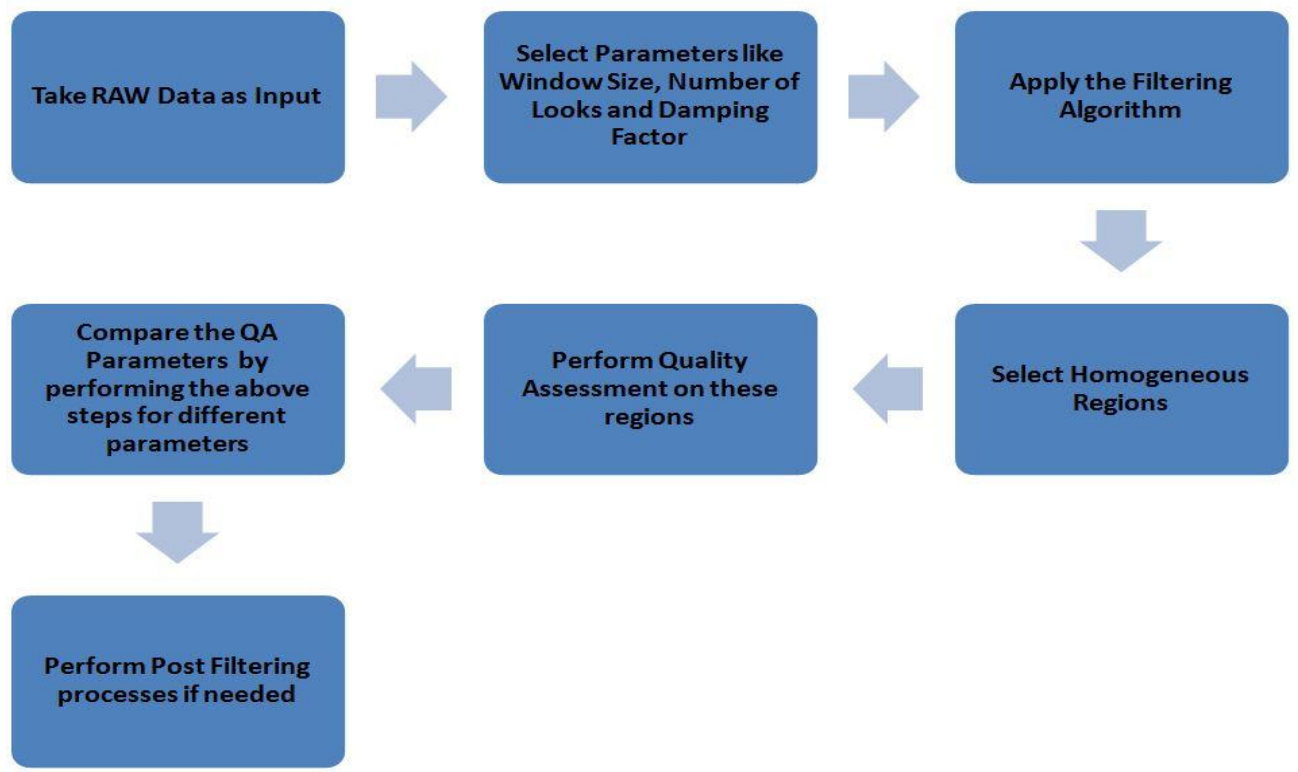

\section{Figure 1}

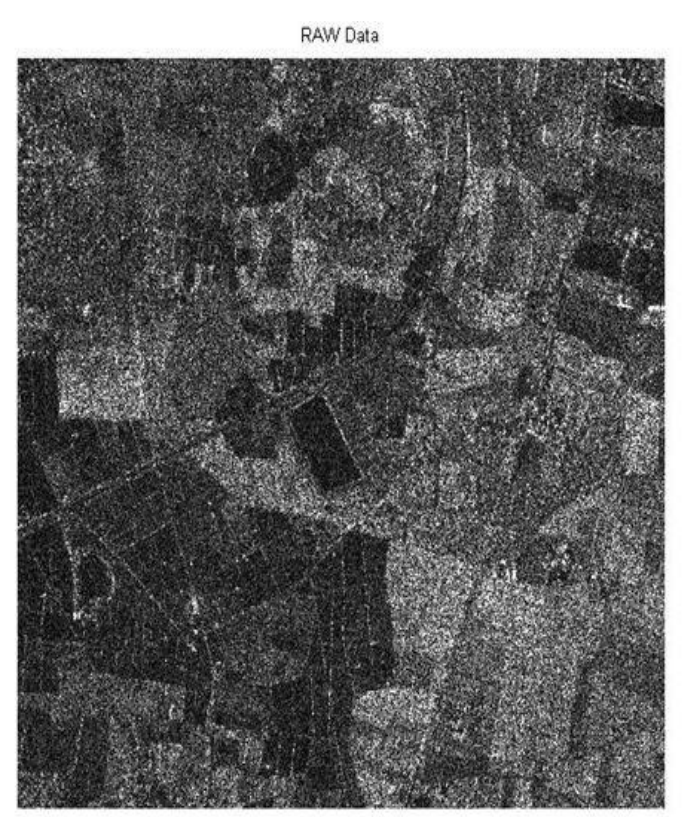

Figure 2

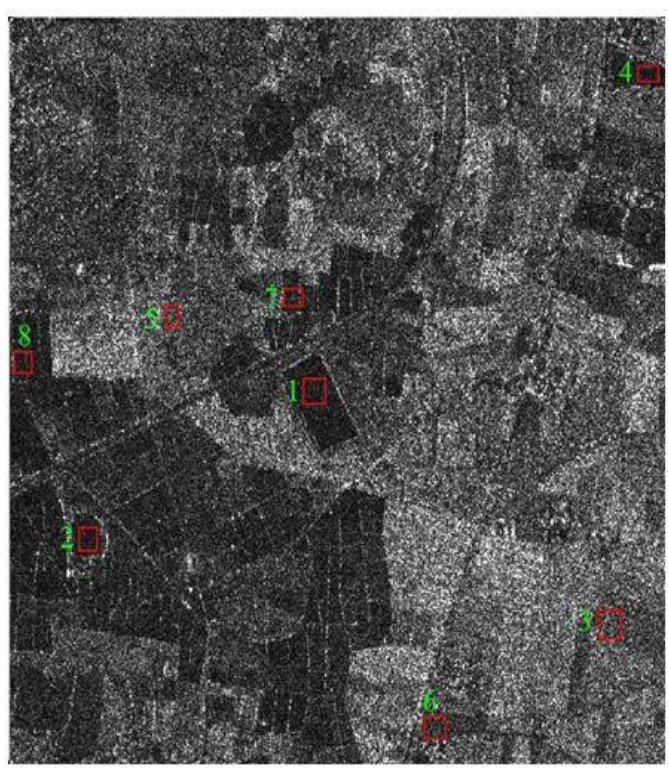

Figure 3

Figure 1 displays the common flow of work done and how the image has been processed and filtered. For testing and analysis, 16X16 smaller homogeneous regions of the 1024X1024 extract of the RISAT data have been considered. There are 8 such $16 \mathrm{X} 16$ pockets and the 3 quality assessment parameters have been used for comparison.

The data set used to analyze and compare the various filters is RISAT-Amplitude image. RISAT-1 stands for RADAR Satellite-1[14]. It carries a SAR payload operating in C band (5.35 GHz). It was successfully launched in 2012[14]. 1024X1024 smaller regions of the entire data set are extracted and the adaptive speckle filtering is performed on these regions. 1 such region has been considered and the corresponding filtered images have been displayed in the results section. Figure 2 displays the extracted 1024X1024 region. This particular extracted region is part of RISAT-1 FRS-1 (3m) data set where FRS stands from Fine Resolution Scan.

Figure 3 shows the pockets which have been considered for quality assessment. The pockets have been chosen such that the radiometric resolution of the pockets of the raw data is similar to each other. 


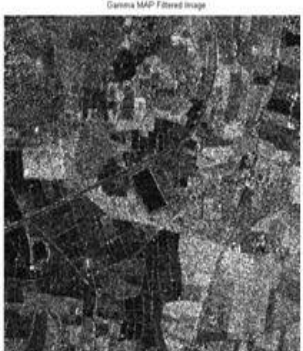

Figure 4.a

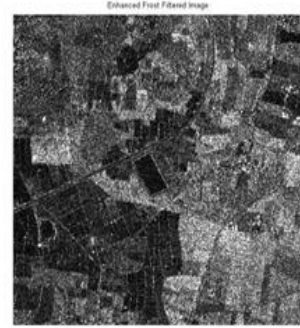

Figure 4.e

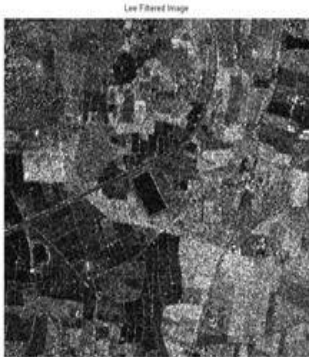

Figure 4.b

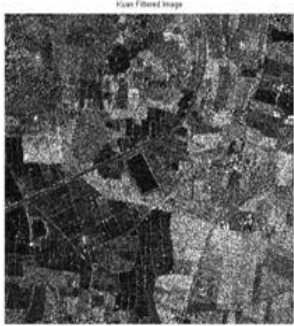

Figure 4.f

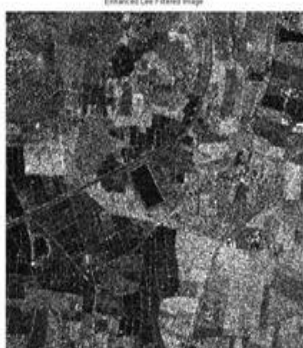

Figure 4.c

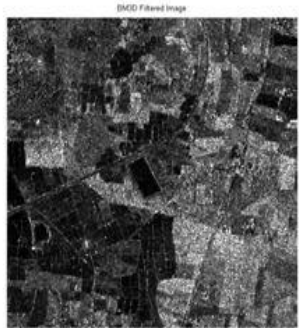

Figure 4.g

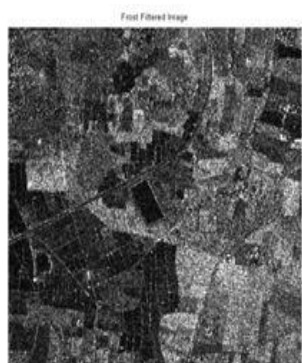

Figure 4.d

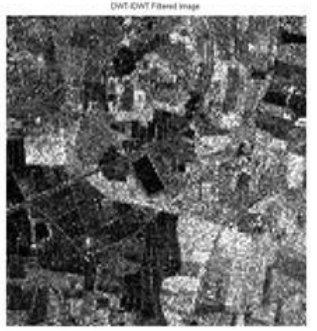

Figure 4.h

The results not only display the filtered images but also the graphs consisting of the quality assessment parameters, thus comparing the performance among the various speckle filters. Figure 3 shows the pockets which have been considered for quality assessment. The pockets have been chosen such that the radiometric resolution of the pockets of the raw data is similar to each other.

Figure 4 shows the filtered outputs. Fig 4.a displays Gamma MAP filter output, Fig 4.b Lee filter output, Fig 4.c Enhanced Lee filter output, Fig 4.d Frost filter output, Fig 4.e Enhanced Frost filter output, Fig 4.f Kuan filter output, Fig 4.g BM3D filter output and lastly Fig 4.h Wavelet Based filter output.

A windows size of $3 \mathrm{X} 3$ and Number of Looks and Damping Factor as 1 has been considered while performing the various filtering operations on the raw data.

Figure 5 displays radiometric resolution as one of the quality assessment parameters. Figure 6 displays the speckle index as the second quality assessment parameter. Figure 7 displays the mean preservation index as the third quality assessment parameter.

The horizontal axis in all 3 figures shows the different 16X16 pockets which have been highlighted in Figure 3. The numbers 1 to 8 in the graphs correspond exactly to the regions which have been shown as in the Figure 3 . The graph of radiometric resolution only contains the value of raw data. Speckle index is computed for filtered regions directly whereas mean preservation index is computed using both the filtered regions as well as the regions in raw data.

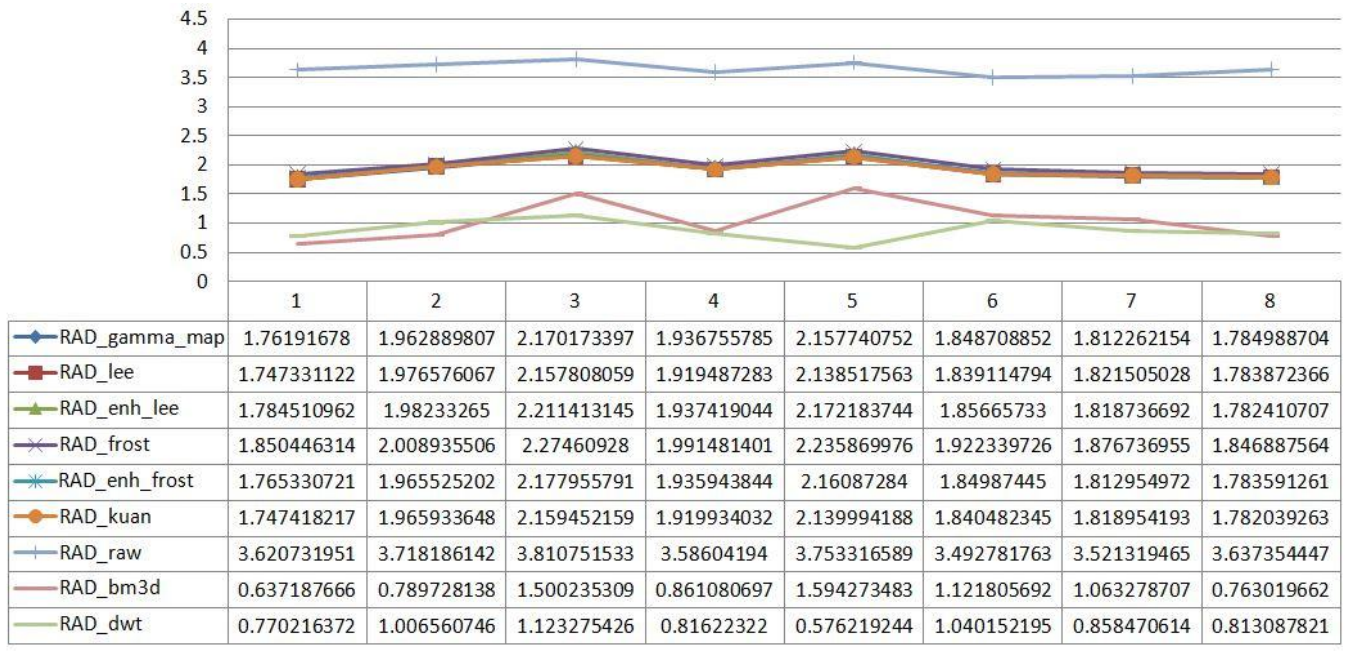

Figure 5 


\begin{tabular}{|c|c|c|c|c|c|c|c|c|}
\hline & \multicolumn{8}{|c|}{$\mathrm{CO}$} \\
\hline & \multicolumn{8}{|c|}{$\mathrm{NON}$} \\
\hline & 1 & 2 & 3 & 4 & 5 & 6 & 7 & 8 \\
\hline$\rightarrow$-SI_gam & 0.224886474 & 53558166 & 2838334 & 49792141 & 281997085 & 23718727 & 0.232006808 & 0.228144408 \\
\hline- -SI_lee & 22831327 & 0.255534941 & 282007019 & 0.247309885 & 0.279162968 & 0.235821481 & 0.233318515 & 0.227986573 \\
\hline$\mp$-SI_enh_lee & 22807686 & 0.256367324 & 0.289943407 & 0.249887579 & 0.284130577 & 0.238319941 & 0.232925499 & 0.227779945 \\
\hline *SI_frost & 237434772 & 0.260221183 & 0.299 & 0.257691339 & 0.293580615 & 0.2477 & 0.241185941 & 0.236927879 \\
\hline *SI_enh_frost & 0 & 67 & 0 & 0.2 & 45 & 0.2 & 0.2321 & 0.227 \\
\hline$\rightarrow-S I$ & 89 & 34 & - & 0.2 & 48 & 07 & 373 & sent \\
\hline$+\mathrm{SI} \_\mathrm{bm} 3 \mathrm{~d}$ & 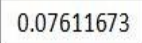 & 76 & & & 1472055 & 0.137863809 & 0.130222465 & 0.091819843 \\
\hline -SI_dwt & 092724847 & 0.122866273 & 0.138056363 & 0.098528076 & 0.068589647 & 0.127217207 & 0.103884234 & 0.098131605 \\
\hline
\end{tabular}

Figure 6

\begin{tabular}{|c|c|c|c|c|c|c|c|c|}
\hline \multirow[t]{2}{*}{$\begin{array}{r}0.08 \\
0.06 \\
0.04 \\
0.02 \\
0\end{array}$} & \multicolumn{8}{|c|}{ 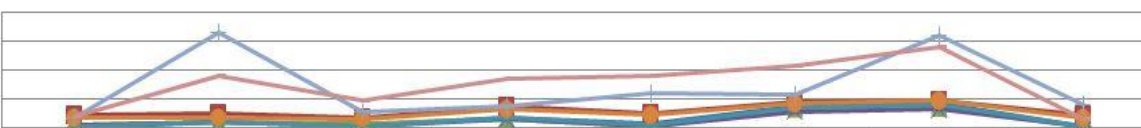 } \\
\hline & 1 & 2 & 3 & 4 & 5 & 6 & 7 & 8 \\
\hline$\rightarrow-$ MPI_gamma_map & 0.000115319 & 003553805 & 0.001662771 & 0.007245484 & 0.002456259 & 0.014498433 & 0.016442521 & 0.001310352 \\
\hline$-\mathrm{C}-\mathrm{MPI}$ lee & 0.00851054 & 0.01032188 & 0.007418518 & 0.014663479 & 0.010067033 & 0.017476489 & 0.019225822 & 0.008542486 \\
\hline$\mp$-MPI_enh_lee & 0.002306379 & 0.003983883 & 0.00245346 & 0.006284348 & 0.000423493 & 0.013416928 & 0.013893112 & 0.001159157 \\
\hline$\asymp$ MPI_frost & 0.001453019 & 0.003508534 & 0.000697666 & 0.005816103 & 0.000423493 & 0.011426332 & 0.012630102 & 0.001159157 \\
\hline *MPI_enh_frost & 0.000438212 & 0.003666984 & 0.000883711 & 0.007072972 & 0.001984367 & 0.014247649 & 0.01597474 & 0.001209555 \\
\hline$\rightarrow-$ MPI_kuan & 0.006550118 & 0.007333967 & 0.006174347 & 0.013012298 & 0.00844566 & 0.01684953 & 0.018641095 & 0.006954944 \\
\hline+ MPI_bm3d & 0.007126712 & 0.065756711 & 0.011406844 & 0.014835991 & 0.0240786 & 0.022931034 & 0.063524734 & 0.015623425 \\
\hline -MPI_dwt & 0.007795563 & 0.035492779 & 0.019430007 & 0.034354437 & 0.035900104 & 0.043197492 & 0.056040229 & 0.006123375 \\
\hline
\end{tabular}

Figure 7

\section{Conclusion}

It can be seen from Figure 4 that visually differentiating the performance of the filters isn't possible. Through the quality assessment parameters it can be observed that the advanced algorithms like BM3D and wavelet based filtering perform comparatively better than the standard adaptive speckle filters in terms of radiometric resolution and speckle index. This is because these filters not only preserve mean but also drastically reduce the standard deviation. Thus the standard deviation of each of the regions is very less compared to the standard deviation of the regions in raw data. A low value of standard deviation drastically improves not only the radiometric resolution but also the speckle index. On the other hand, the mean preservation index of the standard speckle filters turn out to be better simply because the mean preservation by these filters is better compared to the advanced speckle filters. The difference between the mean values of the regions in the filtered data and the mean values of the regions in the raw data is lesser in case of the standard speckle filters. Thus their MPI values turn out to be better compared to BM3D and wavelet based filtering.

The standard speckle filters as well as the advanced filters are used extensively to minimize speckle noise. While standard speckle filters have the advantage of fast computation, they are unable to reduce speckle noise that effectively as the advanced filters. On the other hand, advanced speckle filters have the disadvantage of time as well algorithm complexity compared to the standard speckle filters. Thus it can be safely concluded that each method has its own pros and cons and thus can be judiciously used as per the application at hand. The radiometric improvement with degradation of geometric features may not be good for applications needing edge or object or feature detection, but are suitable for those needing classification of broad features such as agricultural crop estimation, forest bio-mass and geological changes.

\section{References}

[1]. D. A. Bhattacharya, "Speckle filtering/speckle statistics," ppt.

[2]. L. Gagnon and A. Jouan, "Speckle filtering of sar images - a comparative study between complex-wavelet-based and standard filters," SPIE Proc. conference Wavelet Applications in Signal and Image Processing V, 1997.

[3]. $\quad$ E. Nezry, "Adaptive speckle filtering in radar imagery," Thesis Paper, pp. 3-56, 2014.

[4]. J. S. Lee, "Digital image enhancement and noise filtering by use of local statistics," IEEE Transactions on Pattern Analysis and Machine Intelligence, vol. PAMI-2, No-2, 1980.

[5]. R. Touzi, "A review of speckle filtering in the context of estimation theory," IEEE Transactions on Geoscience and Remote Sensing, vol. 40, pp. 2392-2404, 2002. 
[6]. A. Misra and B. Kartikeyan, "Denoising techniques for synthetic aperture radar data a review," International Journal of Computer Engineering and Technology (IJCET), vol. 6, pp. 1-11, 2015.

[7]. Y. Huang and J. L. van Genderen, "Evaluation of several speckle filtering techniques for ers-1 and 2 imagery," International Archives of Photogrammetry and Remote sensing, vol. XXXI, Part B2. Vienna, 1996.

[8]. P. A. Hatwar and D. H. R. Kher, "Analysis of speckle noise reduction in synthetic aperture radar images," International Journal of Engineering Research and Technology (IJERT), vol. 4, pp. 508-512, 2015.

[9]. K. Dabov, A. Foi, V. Katkovnik, and K. Egiazarian, "Image denoising by sparse 3 d transform-domain collaborative filtering," IEEE Transactions on Image Processing, vol. 16, pp. 1-16, 2007.

[10]. M.Lebrun, "An Analysis and implementation of the bm3d image denoising method," Image Processing On Line, vol. 2, pp. 175$213,2012$.

[11]. A. Misra and B. Kartikeyan, "Denoising of sar imagery in the wavelet framework: Performance analysis," International Journal of Remote Sensing and Geoscience (IJRSG), vol. 3, pp. 1-11, 2014.

[12]. S. Dellepiane and E. Angiati, "Quality assessment of despeckled sar images," IEEE Journal of Selected Topics in Applied Earth Observations and Remote Sensing, vol. 7, pp. 691-707, 2014.

[13]. J. M. Martinez, J. Mittermayer, and M. R. Cassola, "Radiometric resolution optimization for future sar systems," IEEE Transactions on Geoscience and Remote Sensing, pp. 1738-1741, 2004.

[14]. RISAT-1, National Remote Sensing Centre Indian Space Research Organization. http://www.nrsc.gov.in/RISAT-1. 